OPEN ACCESS

Edited by:

Felipe Klein Ricachenevsky, Federal University of Rio Grande, Brazil

Reviewed by:

Marta Silva Lopes,

Institute of Agrifood Research and

Technology (IRTA), Spain

Dawei Xin,

Northeast Agricultural University,

China

*Correspondence:

Hui Liu

hui.liu@uwa.edu.au

Guijun Yan

guijun.yan@uwa.edu.au

Specialty section:

This article was submitted to

Plant Abiotic Stress,

a section of the journal

Frontiers in Plant Science

Received: 11 May 2020

Accepted: 11 August 2020

Published: 28 August 2020

Citation:

Lu L, Liu H, Wu Y and Yan G (2020)

Development and Characterization of Near-Isogenic Lines Revealing

Candidate Genes for a Major

7AL QTL Responsible for

Heat Tolerance in Wheat.

Front. Plant Sci. 11:1316.

doi: $10.3389 /$ fpls.2020.01316

\section{Development and Characterization of Near-Isogenic Lines Revealing Candidate Genes for a Major 7AL QTL Responsible for Heat Tolerance in Wheat}

\author{
Lu Lu ${ }^{1,2,3}$, Hui Liu ${ }^{1,2^{*}}$, Yu Wu ${ }^{3}$ and Guijun Yan ${ }^{1,2^{*}}$ \\ ${ }^{1}$ Faculty of Science, UWA School of Agriculture and Environment, The University of Western Australia, Perth, WA, Australia, \\ 2 The UWA Institute of Agriculture, The University of Western Australia, Perth, WA, Australia, ${ }^{3}$ Chengdu Institute of Biology, \\ Chinese Academy of Sciences, Chengdu, China
}

Wheat is one of the most important food crops in the world, but as a cool-season crop, it is more prone to heat stress, which severely affects crop production and grain quality. Heat tolerance in wheat is a quantitative trait, and the genes underlying reported quantitative trait loci (QTL) have rarely been identified. Near-isogenic lines (NILs) with a common genetic background but differing at a particular locus could turn quantitative traits into a Mendelian factor; therefore, they are suitable material for identifying candidate genes for targeted locus/loci. In this study, we developed and characterized NILs from two populations Cascades $\times$ Tevere and Cascades $\times$ W156 targeting a major 7AL QTL responsible for heat tolerance. Molecular marker screening and phenotyping for SPAD chlorophyll content and grain-yield-related traits confirmed four pairs of wheat NILs that contrasted for heat-stress responses. Genotyping the NILs using a 90K Infinium iSelect SNP array revealed five single nucleotide polymorphism (SNP) markers within the QTL interval that were distinguishable between the isolines. Seven candidate genes linked to the SNPs were identified as related to heat tolerance, and involved in important processes and pathways in response to heat stress. The confirmed multiple pairs of NILs and identified candidate genes in this study are valuable resources and information for further fine-mapping to clone major genes for heat tolerance.

Keywords: heat tolerance, near-isogenic lines, 7AL, quantitative trait loci, single nucleotide polymorphism assay, candidate genes, wheat

\section{INTRODUCTION}

Crop growth and productivity are often limited by abiotic stresses, especially heat and drought (Priya et al., 2019). Wheat is one of the most important food grain crops in the world, but being a cool-season crop, it often experiences heat stress. Each $1^{\circ} \mathrm{C}$ rise in temperature above the optimum can cause a 3-5\% reduction in single grain weight under controlled environments (Dawson and Wardlaw, 1989) or field conditions (Wiegand and Cuellar, 1981). Phenology is known to confound 
crop responses to heat; therefore, the effect of heat stress depends on its timing, duration, and frequency (Rezaei et al., 2015; Balla et al., 2019). While conventional breeding has developed some heat-tolerant lines, it is a time-consuming process, and the genetic and physiological basis of the improvements remain unclear (Driedonks et al., 2016). Understanding the underlying mechanism of heat tolerance and identifying candidate genes will help to accelerate the breeding of heat-stress-resilient genotypes (Budak et al., 2015).

Heat tolerance is a quantitative trait (Moffatt et al., 1990a; Yang et al., 2002) that involves complex genetic, physiological, and biochemical controls and is affected by environmental factors. Numerous heat tolerance QTL have been identified; for example, Yang et al. (2002) found QTL on the short arms of chromosomes $1 \mathrm{~B}$ and 5A linked to grain filling duration; Mason et al. (2010, 2011) reported several QTL for heat susceptibility indices and yield traits on chromosomes $1 \mathrm{~A}, 1 \mathrm{~B}, 2 \mathrm{~A}, 2 \mathrm{~B}, 3 \mathrm{~B}, 5 \mathrm{~A}$, and $6 \mathrm{D}$; Paliwal et al. (2012) reported QTL on chromosomes 2B, 7B, and $7 \mathrm{D}$ for thousand-grain weight, grain fill duration, and canopy temperature depression, respectively; Vijayalakshmi et al. (2010) reported QTL on chromosomes 2A, 3A, 4A, 6A, 6B, and 7A with significant effects on grain yield, grain weight, grain filling, stay green, and senescence-associated traits under post-anthesis hightemperature stress in wheat. Most of these reported QTL have been based on mapping using low-density simple sequence repeat (SSR) markers and/or amplified fragment length polymorphism (AFLP) markers. Talukder et al. (2014) increased the marker density to 972 molecular markers and identified QTL associated with different traits related to heat tolerance in wheat. They found that QTL QHtscc.ksu-7A on chromosome 7A was consistently identified for traits thylakoid membrane damage (TMD), plasma membrane damage (PMD), and SPAD chlorophyll content (SCC), with high logarithm of odds (LOD) values ranging from 4.15 to 6.95 and explaining high phenotypic variations ranging from 18.9 to $33.5 \%$. This major QTL QHtscc.ksu-7A, with flanking markers Xbarc121 and Xbarc49, was chosen as the target locus for developing NILs in this study.

It remains challenging to use QTL markers directly in breeding programs due to the large genomic intervals of the most identified QTL (Mia et al., 2019). One solution for identifying candidate genes and closely linked markers is to develop NILs, which turn quantitative traits into Mendelian factors (Liu et al., 2006). NILs are pairs of lines that have the same genetic background between isolines, except for the targeted locus (Dorweiler et al., 1993). NILs make it easier to study phenotypic impacts attributable to a specific gene or locus (Pumphrey et al., 2007). Characterizing NILs through genotypephenotype association analyses can lead to the identification of candidate genes (Mirdita et al., 2008; Liu et al., 2010; Mia et al., 2019; Wang et al., 2019). Traditionally, NILs development has been considered time-consuming and tedious (Tuinstra et al., 1997). By combining a fast generation cycling system (FGCS) (Zheng et al., 2013) with heterogeneous inbred family (HIF) method and repeated DNA marker-assisted selection (MAS) (Tuinstra et al., 1997), the NIL development process can be shortened to about six generations per year (Yan et al., 2017).
Single nucleotide polymorphism (SNP) markers are highdensity DNA markers widely used in genetic studies, including genetic diversity, phylogenetic relationships, and marker-trait associations, such as genome-wide association study (GWAS) or QTL mapping (Wang et al., 2014; Cabral et al., 2018). The 90k SNP array, developed from hexaploid wheat and Aegilops tauschii sequences (Wang et al., 2014) with dense coverage of the wheat genome, has been extensively harnessed for genetic research (Cavanagh et al., 2013; Avni et al., 2014; Colasuonno et al., 2014; Russo et al., 2014; Sukumaran et al., 2015). Due to its efficiency of characterizing genetic resources and discriminating between closely related lines (Rimbert et al., 2018), 90k SNP genotyping was used in this study to characterize the developed NILs, in combination with phenotyping under controlled environments.

The objectives of this study were to 1 ) develop and confirm NILs targeting the major heat tolerance QTL on chromosome $7 \mathrm{~A}, 2)$ identify candidate gene(s) underlying the 7A QTL responsible for heat tolerance by genotypic and phenotypic characterization of the NILs, and 3) shed light on the genetic mechanism of heat tolerance in wheat by inspecting this major genomic region and investigating its underlying candidate genes.

\section{MATERIALS AND METHODS}

\section{Plant Materials and Selection of Crossing Parents}

In a previous study, 499 wheat genotypes from a variety of sources were screened and evaluated for heat-stress responses (Hameed, 2015). Among them, cultivars Tevere and W156 showed heat tolerance at both the seedling and reproductive stages with high yield, whereas Cascades (Aroona//Tadorna/Inia 66) was sensitive at both stages (unpublished data). Cascades and Tevere are two common wheat cultivars and W156 is a landrace, which originated from Australia, Italy, and India, respectively. When the flanking marker Xbarc49 of the targeted 7A QTL was used for genotyping the three cultivars, heat-tolerant Tevere and W156 showed the tolerance allele at $216 \mathrm{bp}$, and heat-susceptible Cascades showed the susceptibility allele at $203 \mathrm{bp}$. The three cultivars were therefore used to establish two cross populations, Cascades/W156 and Cascades/Tevere, for the development of NILs targeting the 7A locus.

\section{Development of NILs}

NILs were developed from the two populations using the HIF method (Tuinstra et al., 1997) in combination with embryoculture-based FGCS (Zheng et al., 2013; Yan et al., 2017), following a similar procedure as described in Wang et al. (2019). Specifically, MAS started from the second generation of progenies (F2) (Figure 1A) where genomic DNA was isolated from two-week-old seedlings of each plant using a modified CTAB method (Murray and Thompson, 1980). Xbarc49 (Figure 1B), the flanking marker of QHtscc.ksu-7A (Talukder et al., 2014), was used to identify heterozygous progenies ( $\mathrm{Rr}$ ) from the two cross populations (Figure 1C). PCR reactions were performed, and amplified products viewed following the 


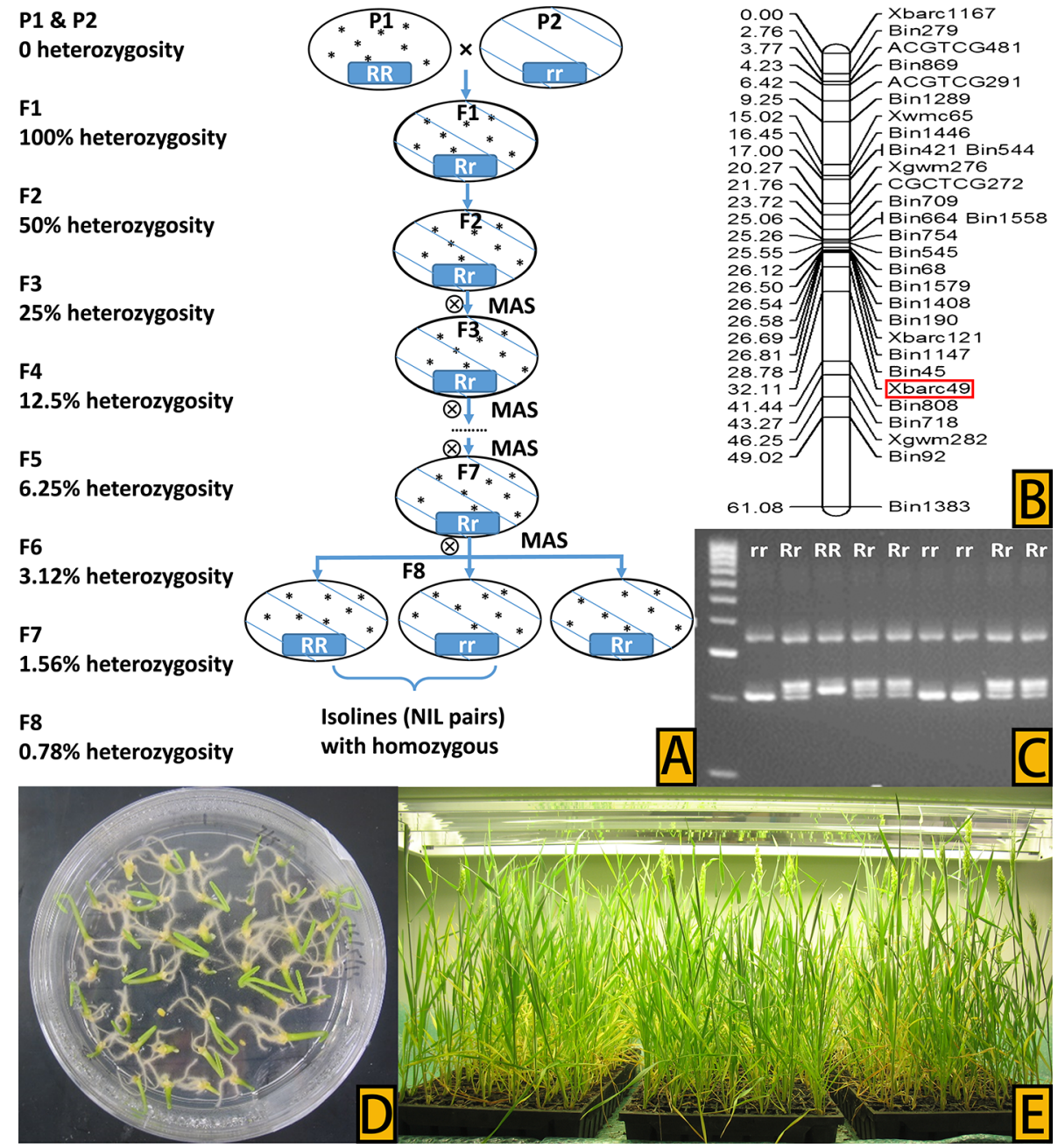

FIGURE 1 | (A) Process of the HIF method to develop NIL pairs, with percentage heterozygosity in each generation shown on the left of the flow chart. (B) QTL hotspot in wheat chromosome 7A and the marker (in the box) used for selection, adapted from Talukder et al., 2014. (C) Marker-assisted selection of different progeny types, with tolerance progeny, susceptible progeny, and heterozygous progeny marked RR, $r$, and Rr, respectively, on the top of the gel bands. (D) Culture of young embryos on Petri plates in a sterile medium. (E) Seedlings from embryo culture growing in the plant growth chamber.

protocol described in Wang et al. (2018). MAS of heterozygous progenies, together with embryo-culture-based FGCS (Figures $1 \mathrm{D}, \mathrm{E})$, continued until the eighth generation of progenies (F8); at F8, only those homozygous progenies from single seed descent with the tolerance allele $(\mathrm{RR} /+)$ from either W156 or Tevere and those with susceptibility allele ( $\mathrm{rr} /-$ ) from Cascades were selected as pairs of candidate NILs. Thirteen pairs of F8 NILs, numbered from NIL1 to NIL13, developed from the two populations were used as putative NIL pairs to screen their phenotypes for performance under heat stress.

\section{Plant Growth Conditions and Heat Treatments}

The seeds of all isolines were germinated in water on Petri dishes, before sowing one plant per pot $(8 \mathrm{~cm} \times 8 \mathrm{~cm} \times 16 \mathrm{~cm})$ containing soil media (5:2:3 compost:peat:sand, $\mathrm{pH}$ 6.0) (Mia et al., 2019). For each isoline, six pots (three replicates each for the control and the heat-stress treatment) were grown in a naturally lit glasshouse at The University of Western Australia, Crawley, Western Australia (31 $59^{\circ}$ ' S, 115²9’E). The plants were fertilized fortnightly with 'Diamond Red' (Campbells Fertilisers Australasia Pty Ltd, Australia) from four weeks after sowing until the end of the grain-filling period. The glasshouse environment (temperature, relative humidity, and light intensity) is detailed in Supplementary Table S1. The experiment was arranged in a completely randomized block design.

Anthesis date, as Zadoks' growth scale Z60 (Zadoks, 1974), of each plant was recorded by tagging each plant on the wheat head where the first anther appeared. The time point to start the heat treatment, treatment temperature, and other settings were as per previous studies (Pradhan et al., 2012; Talukder et al., 2014; Shirdelmoghanloo et al., 2016a). Specifically, on the 10th day after anthesis (DAA), the three treatment pots were moved into a growth chamber set to $37 / 27^{\circ} \mathrm{C}$ (day/night), 14-h photoperiod, 
and $420 \mu \mathrm{mol} \mathrm{m} \mathrm{m}^{-2} \mathrm{~s}^{-1}$ light intensity for the 3-day heat treatment. Enough water was given to the plants to ensure there was no drought stress, only heat stress. The pots were returned to the glasshouse after the heat treatment.

\section{Phenotype Screening}

Chlorophyll contents were measured on flag leaves using a handheld portable chlorophyll meter (SPAD-502Plus; Konica Minolta, Osaka) for both the control and heat-stressed plants. Time points for measurements followed the procedures described in Pradhan et al. (2012). Changes in SPAD chlorophyll contents $(\triangle \mathrm{SCC})$ in flag leaves were calculated based on differences in chlorophyll contents before (as 'Chl10' at 10 DAA) and after (as 'Chl13' at 13 DAA) treatment, as follows: $\triangle \mathrm{SCC}=$ mean of Chl10 mean of Chl13 (Shirdelmoghanloo et al., 2016b). Measurements and calculations were the same for controls.

Agronomic traits (grain number and grain yield per plant) were measured after harvest for plants in the control and heatstressed treatments. The performance differences in final yield between isoline pairs were determined by subtracting mean values in the control from those in the heat treatment.

Statistical analyses were undertaken using t-tests to compare phenotypic variation in the NIL pairs. True NILs were confirmed if significant differences existed in the performance of tested traits between isolines, and their resistant and susceptible phenotypes matched their genotypes of Xbarc49's RR/+ and rr/ - alleles, respectively.

\section{Genotyping by 90k Infinium iSelect SNP Array}

The 90K SNP array was used for genotyping, with genotypephenotype associations used to identify candidate gene(s) (Alaux et al., 2018). Specifically, genomic DNA samples of the confirmed NILs were genotyped using the Wheat 90K Illumina iSelect Array (Wang et al., 2014). SNP clustering and genotype calling were performed using GenomeStudio 2.0 software (Illumina). SNPs with a call frequency $<0.8$ (i.e., missing data points $>20 \%$ ), minor allele frequency (MAF) $<0.05$ or heterozygous calls $>0.25$ were removed. SNP sequences that differed between NIL pairs were used to perform a BLAST search against the wheat reference genome (IWGSC, 2018). SNPs located on the 7AL chromosome arm, especially those within the marker interval of QHtscc.ksu-7A (Talukder et al., 2014), were scrutinized using JBrowse (http://www.wheatgenome.org/Tools-and-Resources/ Sequences) for candidate gene discovery.

\section{RESULTS}

\section{Four NIL Pairs Confirmed With Significant Differences in Chlorophyll Content}

After comparing the $\triangle$ SCC data for NIL pairs, no significant differences were observed among the 13 putative NIL pairs grown in the non-stressed (control) treatment, whereas the isolines of four NIL pairs significantly differed in the heatstressed treatment. Of these, the isolines with positive alleles $(+$ NILs) had smaller reductions in SPAD chlorophyll content than isolines with negative alleles (-NILs). The isolines of NILs 5, 10 , and 13 differed significantly at $\mathrm{P}<0.05$, and NIL 9 differed significantly at $\mathrm{P}<0.01$ (Table $\mathbf{1}$ ).

\section{Tolerant Isolines Performed Better Than Susceptible Isolines on Other Agronomic Traits in the Confirmed NILs}

Further investigation showed that significant differences existed between most of the treated isolines and their corresponding controls for yield-related traits. The differences or gaps in grain number per pot and yield per pot between the control and heatstressed treatments are shown in Figure 2 to compare yield performance in tolerant and susceptible isolines. For grain number, three of the four +NILs $(5,9$, and 10) had smaller gaps than -NILs. For yield, all four +NILs had smaller gaps between non-stressed and stressed treatments than their counterparts. Generally, heat stress had smaller negative effects on grain number and yield in +NILs than -NILs.

\section{Five SNP Markers With Consistent Contrasting Genotypes in the Confirmed NIL Pairs}

Of the 81,587 SNPs on the array, 53,052 were analyzed across the 21 chromosomes after removing those that did not meet the

TABLE 1 | Reduction of SPAD chlorophyll content $(\triangle S C C)$ in the confirmed NIL pairs.

\begin{tabular}{|c|c|c|c|c|c|}
\hline \multirow[t]{2}{*}{ NIL name } & \multirow[t]{2}{*}{ Population } & \multicolumn{4}{|c|}{$\Delta S C C$ (Chl10-Chl13) } \\
\hline & & \multicolumn{2}{|c|}{ Control } & \multicolumn{2}{|c|}{ Treatment } \\
\hline NIL5(+) & Cascade/Tevere & -0.4 & ns & 0.53 & * \\
\hline NIL5(-) & & -0.8 & & 7.13 & \\
\hline NIL9(+) & Cascade/W156 & 1.1 & ns & 4.57 & ** \\
\hline NIL10(-) & & 0.6 & & 12.23 & \\
\hline NIL13(+) & Cascade/Tevere & 0.15 & ns & 1.7 & * \\
\hline NIL13 (-) & & 1.27 & & 6.7 & \\
\hline
\end{tabular}

ns = non-significant at $P \leq 0.05 ;{ }^{*}=$ significant at $P \leq 0.05 ;{ }^{* *}=$ significant at $P \leq 0.01$. The statistics was done using $t$-test. (+) indicates isolines with resistance allele from W156 or Tevere; $(-)$ indicates isolines with susceptibility allele from Cascades. 

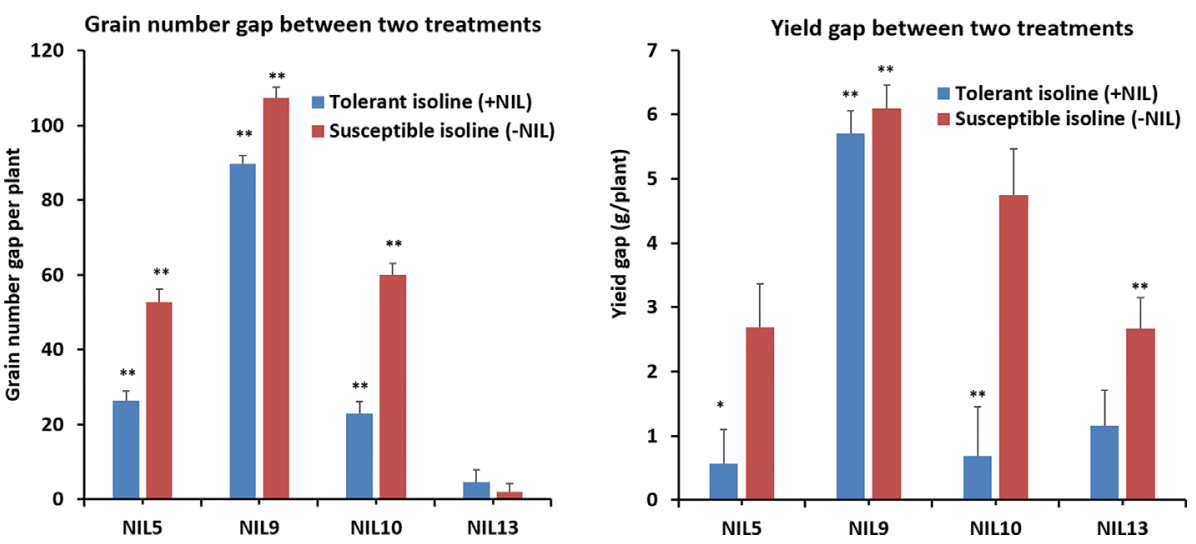

FIGURE 2 | Differences in agronomic trait gaps between the confirmed isolines of tolerant and susceptible alleles. Three replicates each for the control (naturally lit glasshouse with conditions described in detail in Table S1) and the heat-stressed treatment (growth chamber set at $37 / 27^{\circ} \mathrm{C}$ day/night) were used for statistical analysis. The column indicates trait gaps between the treatment and control, with error bar on top. * indicates significant difference at $\mathrm{P} \leq 0.05$; ** indicates significant difference at $\mathrm{P} \leq 0.01$. Statistics done using t-test.

selection criteria. Analyzing the SNPs among the four confirmed pairs of isolines identified five SNPs within the 7AL QTL region with consistent contrasting genotypes between the resistant and susceptible isolines (Table 2).

\section{Candidate Genes Identified by Blasting the Wheat Reference Genome}

Seven candidate genes were identified within the QHtscc.ksu-7A region by blasting the above five SNP markers with wheat reference genome RefV1.0. The annotations for high and low confidence genes of RefV1.0 were used, with various databases compared, including NCBI (https://www.ncbi.nlm.nih.gov) and InterMine (http://www.wheatgenome.org/Tools-and-Resources/ Sequences), to determine possible gene functions (Table 3).

Markers BS00071558_51 and wsnp_Ku_c5160_9203226 were co-located on gene TraesCS7A01G612600LC, with their SNP variations in the intron and untranslated region (UTR), respectively (Figure 3). Markers wsnp_Ku_rep_c113718_96236830 and wsnp_RFL_Contig2864_2688208 were co-located on gene TraesCS7A01G432000 and TraesCS7A01G431600, respectively, with their SNP variations in the UTR and exon regions. Although no gene was co-located on wsnp_Ra_c26491_36054023, the marker was located close to genes TraesCS7A01G428200 (15,752 bp away) and TraesCS7A01G428400 (273,590 bp away), both of which function as peroxidase.

Blasting the sequence of TraesCS7A01G612600LC in NCBI identified five ESTs related to plant biotic or abiotic stress responses (Manickavelu et al., 2012). Genes TraesCS7A01G432000 and TraesCS7A01G431600 were involved in important biochemistry or molecular functions, such as protein binding, Zinc finger, F-box domain, Kelch motif, and Per-Arnt-Sim (PAS) domain, all of which are involved in various pathways of plant responses and reactions to external stimulus (Craig and Tyers, 1999; Adams et al., 2000; Prag and Adams, 2003; Hefti et al., 2004; van den Burg et al., 2008; Hennig et al., 2009; Moglich et al., 2009; Peng et al., 2012; Liu et al., 2015).

Apart from these genes, two other noteworthy genesTraesCS7A01G430500, which functions as a sugar transporter family protein, and TraesCS7A01G430600, which functions as a heat shock protein (HSP) -were located in the interval between marker wsnp_Ra_c26491_36054023 and wsnp_RFL_Contig2864_2688208.

\section{DISCUSSION}

In this study, we reported the development of 13 putative NIL pairs targeting a major locus for heat tolerance. Among them, four pairs were confirmed as true NILs by genotype-phenotype association analysis. The confirmed NILs showed differential responses under non-stressed and heat-stressed conditions. NILs with alleles from the heat-tolerant parents (Tevere and W156) performed better than their counterparts in terms of physiological and agronomical traits, such as chlorophyll content, grain number, and grain yield. Characterization of these NIL pairs revealed that the presence of the tolerance allele significantly increased heat tolerance in the

TABLE 2 | SNPS showing consistent contrast callings in the confirmed four pairs of NILS.

\begin{tabular}{|c|c|c|c|}
\hline No. & Marker Name & SNP & Physical location \\
\hline 1 & BS00071558_51 & {$[\mathrm{T} / \mathrm{C}]$} & chr7A:626897156.626897256 \\
\hline 2 & wsnp_Ku_c5160_9203226 & {$[\mathrm{T} / \mathrm{C}]$} & chr7A:626897816.626898016 \\
\hline 3 & wsnp_Ku_rep_c113718_96236830 & {$[\mathrm{A} / \mathrm{G}]$} & chr7A:625739519.625739719 \\
\hline 4 & wsnp_Ra_c26491_36054023 & {$[\mathrm{A} / \mathrm{G}]$} & chr7A:621582917.621583094 \\
\hline 5 & wsnp_RFL_Contig2864_2688208 & {$[\mathrm{T} / \mathrm{C}]$} & chr7A:625640069.625640169 \\
\hline
\end{tabular}


TABLE 3 | Function annotations of candidate genes.

\begin{tabular}{|c|c|c|c|c|}
\hline Gene & Physical position & Database & Identifier & Description \\
\hline TraesCS7A01G612600LC & 626894752.626898021 & EMBL-EBI & $\begin{array}{l}\text { BQ245642 } \\
\text { HX055146 } \\
\text { HX055177 } \\
\text { CJ956871 } \\
\text { CJ945027 }\end{array}$ & $\begin{array}{l}\text { Yield improvement under stress } \\
\text { response to blast fungus } \\
\text { wheat responses to fungi infections } \\
\text { response to powdery mildew infection } \\
\text { responses to fungi infections }\end{array}$ \\
\hline TraesCS7A01G432000 & 625737761.625740656 & Interpros & IPR013083 & Zinc finger, RING/FYVE/PHD-type \\
\hline TraesCS7A01G431600 & 625635655.625640991 & $\begin{array}{l}\text { GOs } \\
\text { Interpros } \\
\text { Pfams }\end{array}$ & $\begin{array}{l}\text { GO:0005515 } \\
\text { IPR001810 } \\
\text { IPR015915 } \\
\text { IPR000014 } \\
\text { IPR011498 } \\
\text { PF13426 } \\
\text { PF13415 } \\
\text { PF07646 } \\
\text { PF13418 } \\
\text { PF12937 }\end{array}$ & $\begin{array}{l}\text { Molecular Function: protein binding } \\
\text { F-box domain } \\
\text { Kelch-type beta propeller } \\
\text { PAS domain } \\
\text { Kelch-repeat type } 2 \\
\text { PAS domain } \\
\text { Galactose oxidase, central domain } \\
\text { Kelch motif } \\
\text { Galactose oxidase, central domain } \\
\text { F-box-like }\end{array}$ \\
\hline TraesCS7A01G428200 & 621564527.621567165 & $\begin{array}{l}\text { GOs } \\
\text { Interpros } \\
\text { Pfams }\end{array}$ & $\begin{array}{l}\text { GO:0020037 } \\
\text { GO:0055114 } \\
\text { GO:0004601 } \\
\text { GO:0006979 } \\
\text { IPR000823 } \\
\text { IPR002016 } \\
\text { IPR010255 } \\
\text { IPR019793 } \\
\text { IPR019794 } \\
\text { PF00141 }\end{array}$ & $\begin{array}{l}\text { MF: heme binding } \\
\text { BP: oxidation-reduction process } \\
\text { MF: peroxidase activity } \\
\text { BP: response to oxidative stress } \\
\text { Plant peroxidase } \\
\text { Haem peroxidase, plant/fungal/bacterial } \\
\text { Haem peroxidase } \\
\text { Peroxidases heam-ligand binding site } \\
\text { Peroxidase, active site } \\
\text { Peroxidase }\end{array}$ \\
\hline TraesCS7A01G428400 & 621856684.621857860 & & & Similar function as gene TraesCS7A01G428200 \\
\hline TraesCS7A01G430500 & 624959655.624961254 & $\begin{array}{l}\text { GOs } \\
\text { Interpros } \\
\text { Pfams }\end{array}$ & $\begin{array}{l}\text { GO:0016021 } \\
\text { GO:0016020 } \\
\text { GO:0055085 } \\
\text { GO:0005215 } \\
\text { GO:0022857 } \\
\text { GO:0022891 } \\
\text { IPR020846 } \\
\text { IPR003663 } \\
\text { IPR005828 } \\
\text { IPR005829 } \\
\text { PF00083 }\end{array}$ & $\begin{array}{l}\text { CC: integral component of membrane } \\
\text { CC: membrane } \\
\text { BP: transmembrane transport } \\
\text { MF: transporter activity } \\
\text { MF: transmembrane transporter activity } \\
\text { MF: substrate-specific transmembrane transporter activity } \\
\text { Major facilitator superfamily domain } \\
\text { Sugar/inositol transporter } \\
\text { Major facilitator, sugar transporter-like } \\
\text { Sugar transporter, conserved site } \\
\text { Sugar (and other) transporter }\end{array}$ \\
\hline TraesCS7A01G430600 & 624965100.624967076 & $\begin{array}{l}\text { Interpros } \\
\text { Pfams }\end{array}$ & $\begin{array}{l}\text { IPR013126 } \\
\text { IPR029047 } \\
\text { IPR029048 } \\
\text { IPR018181 } \\
\text { PF00012 }\end{array}$ & $\begin{array}{l}\text { Heat shock protein } 70 \text { family } \\
\text { Heat shock protein } 70 \mathrm{kD} \text {, peptide-binding domain } \\
\text { Heat shock protein } 70 \mathrm{kD} \text {, C-terminal domain } \\
\text { Heat shock protein } 70 \text {, conserved site } \\
\text { Hsp70 protein }\end{array}$ \\
\hline
\end{tabular}

plants. One reason for the higher grain yield and grain number in the heat-tolerant NILs may be the positive correlation between chlorophyll content and gas exchange parameters reported in several studies (Chen et al., 2016; Wang et al., 2016).

Many physiological traits have been closely associated with heat response, such as canopy temperature, leaf senescence, night respiration, chlorophyll fluorescence, and cell membrane thermo-stability (Narayanan, 2018). The original QTL targeted in this study was associated with multiple traits, including thylakoid membrane damage (TMD), plasma membrane damage (PMD), and SPAD chlorophyll content (SCC) (Talukder et al., 2014). Membrane thermostability has a strong genetic correlation with grain yield in wheat (Reynolds et al., 1994; Fokar et al., 1998). Loss of chlorophyll content during grain filling has been associated with reduced yield under field conditions (Reynolds et al., 1994). TMD and PMD have also been associated with grain yield (Moffatt et al., 1990b; Saadall et al., 1990; Reynolds et al., 1994; Araus et al., 1998; Marcum, 1998; Blum et al., 2001; Wahid and Shabbir, 2005). Strong correlations among these traits suggest that these traits are under pleiotropic genetic control.

Leaf chlorophyll content is a major indicator of the photosynthetic capability of plant tissues (Rao et al., 2001; Pietrini et al., 2017). Some studies that focused on yield and photosynthetic traits (Raven and Griffiths, 2015; Gaju et al., 2016; Merchuk-Ovnat et al., 2016) have shown that the photosynthetic function duration of leaves is closely correlated to grain yield in wheat. Furthermore, spectral characteristics measured by SPAD are a good indicator for evaluating crop responses to high 


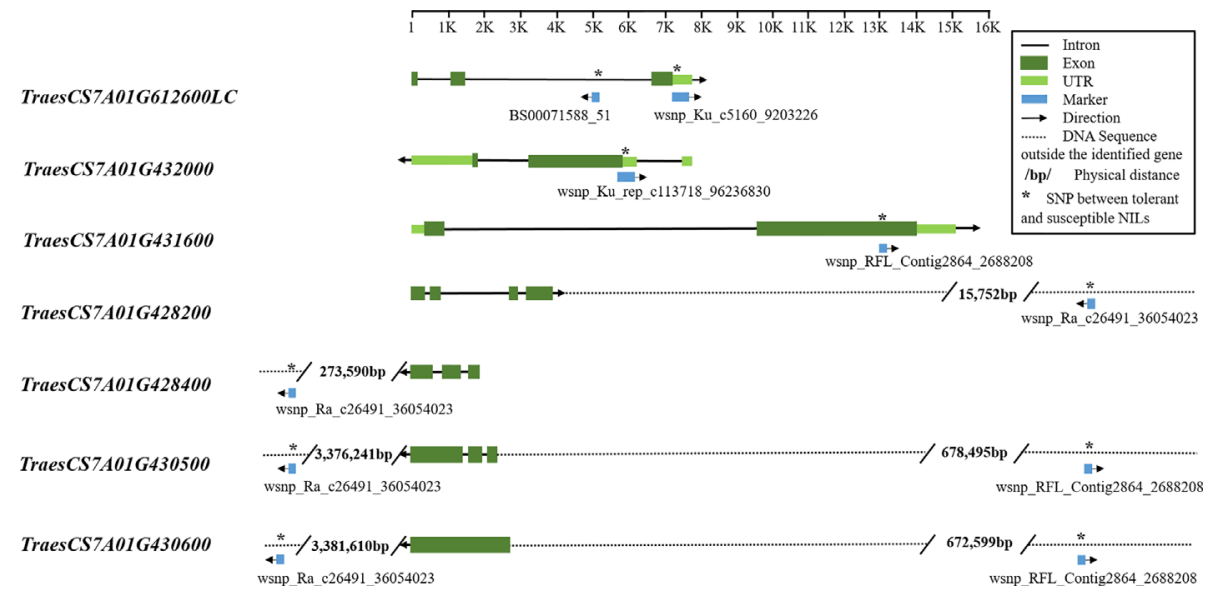

FIGURE 3 | Candidate gene structures and SNPs between tolerant and susceptible NILs. The structural information of genes and SNP markers was extracted from the wheat genome database (https://urgi.versailles.inra.fr/jbrowseiwgsc/gmod_jbrowse). The SNP positions between tolerant and susceptible NILs are marked with an asterisk. For the first three genes, SNPs distinguishing tolerant and susceptible NILs were within the identified genes, either exon, intron, or untranslated region (UTR); for the other four genes, SNPs were outside the candidate genes and their physical distances to the genes are labeled.

temperature (Talukder et al., 2014; Tao et al., 2016). Due to its correlation with yield and other performance indicators under heat stress, chlorophyll content measured by SPAD could be used as an easy and reasonable morphological marker for assessing heat tolerance, especially at the initial mass screening stage. The smaller the reduction in SPAD chlorophyll content ( $\triangle \mathrm{SCC}$ ), the more tolerant the plant should be to heat stress. SPAD meters have been used to estimate leaf chlorophyll content in research and agricultural practices because it is a quick, simple and non-destructive method (Novichonok et al., 2016; Padilla et al., 2018; Zhao et al., 2018; de Souza et al., 2019; Galanti et al., 2019; Zhang et al., 2019). Here, agronomic traits such as grain number and grain yield were also measured to further confirm the true NIL pairs, which are valuable material for further studies of post-anthesis heat tolerance in wheat.

Except for the report by Talukder et al. (2014), the major QTL QHtscc.ksu-7A targeted in this study has been identified as responsible for heat tolerance in other studies. Vijayalakshmi et al. (2010) reported a 7A QTL linked to marker Xbarc121 for heattolerance traits, including $\mathrm{Fv} / \mathrm{Fm}$ and time to maximum rate of senescence. Talukder et al. (2014) revealed that several ESTs, located in the same wheat deletion bin as Xbarc49, were related to stress response in different studies; therefore, the authors proposed that the major QTL QHtscc.ksu-7A was a genomic region rich in genes related to stress response. Dixit et al. (2015) hypothesized that multiple genes underpinned large-effect QTL. In this study, seven candidate genes within the targeted major 7AL QTL were identified as responsible for heat tolerance post-anthesis. The gene TraesCS7A01G612600LC is a homologous gene to TraesCS7A01G612600L; blasting TraesCS7A01G612600L by Blastx (translated nucleotide to protein) on NCBI with criteria of percentage identity $\geq 50 \%$ and e-value of $<1$ e- 5 revealed its origin from Triticum urartu, and it has been up-regulated at $24 \mathrm{~h}$ osmotic stress in the ABA-dependent signaling pathway (Li et al., 2019).
Therefore, the gene TraesCS7A01G612600LC identified in this study supposedly has a similar function as its homologous gene. Abscisic acid (ABA) is generally considered as a stress signaling hormone, and the expression of stress-responsive genes in plants is primarily regulated by $\mathrm{ABA}$-dependent and $\mathrm{ABA}$-independent pathways (Agarwal and Jha, 2010; Yoshida et al., 2014). The ABAdependent pathway is central to osmotic-stress responses in plants (Li et al., 2019). Moreover, five expression sequence tag (EST) markers associated with TraesCS7A01G612600LC were related to plant biotic or abiotic stress responses. Among these EST markers, HX055146, HX055177, CJ956871, and CJ945027 were related to wheat responses to fungi infections, including Fusarium head blight and powdery mildew (Manickavelu et al., 2012), while BQ245642 encoded a polypeptide useful for yield improvement by improving plant growth and development under at least one stress condition (Kovalic et al., 2007).

The annotation of genes TraesCS7A01G432000 and TraesCS7A01G431600 revealed their involvement in some important biochemistry or molecular functions, such as protein binding, Zinc finger, F-box domain, Kelch motif, PAS domain, and galactose oxidase. F-box domain genes are related to plant resistance, while F-box proteins are associated with cellular functions, such as signal transduction and regulation of the cell cycle during plant vegetative and reproductive growth and development (Craig and Tyers, 1999). For example, F-box protein FOA1 plays a role in ABA signaling involved in seed germination (Peng et al., 2012), ACRE189/ACIF1 regulates cell defense and death when tomato and tobacco are attacked by pathogens (van den Burg et al., 2008), Kelch motifs and kelch-repeat $\beta$-propellers undergo a variety of binding interactions with other molecules (Adams et al., 2000; Prag and Adams, 2003), and a PAS domain acts as a molecular sensor (Hennig et al., 2009; Moglich et al., 2009; Liu et al., 2015) and has been deemed as the key structural motif involved in proteinprotein interactions during physiological reactions (Hefti et al., 
2004). In summary, all these biological structures are extensively involved in various pathways of plant responses and reactions to external stimuli such that we can deduce that genes TraesCS7A01G432000 and TraesCS7A01G431600 regulate heat tolerance by controlling protein structures and protein binding on the biological structures mentioned above to achieve signal transduction, which is the key part in the pathway of heat tolerance.

The remaining four genes TraesCS7A01G428200, TraesCS7A01G428400, TraesCS7A01G430500, and TraesCS7A01G430600 have functions as peroxidase, peroxidase, sugar transporter family protein, and HSP, respectively. The sugar transporter family protein is related to yield as sugar transport is one of the most important processes for plant development and their responses to biotic and abiotic factors (Lalonde et al., 2004; Lemoine et al., 2013). Tolerance to heat stress is frequently associated with maintaining sugar content in source leaves (Vasseur et al., 2011; Zhou et al., 2017). For example, a heat-tolerant tomato (Solanum lycopersicum) genotype had significantly higher fructose and sucrose contents in mature leaves than a heat-sensitive genotype under heat stress (Zhou et al., 2017). Peroxidase is related to stress tolerance-peroxidase activities increased significantly in a heattolerant wheat genotype in response to heat treatment (Sairam et al., 1998). Exposure to heat stress often leads to the generation of destructive ROS (reactive oxygen species), but plants have antioxidant mechanisms to escape excessive ROS. Several studies have shown that peroxidase plays an important role in antioxidant mechanisms and ameliorates the effects of heat stress in wheat (Suzuki et al., 2011; Caverzan et al., 2016). HSPs play a pivotal role as chaperones in conferring biotic and abiotic stress tolerance (Baniwal et al., 2004). The expression of HSP genes induced by high temperature can preserve the stability and function of intracellular proteins and protect them from denaturation through protein folding. They enhance membrane stability and detoxify ROS by positively regulating the antioxidant enzyme system. Additionally, HSP genes use ROS as a signal to molecules to induce HSP production. HSP also enhances plant immunity by accumulating and stabilizing pathogenesis-related proteins under various biotic stresses (Haq et al., 2019). Genotypes generating HSPs can withstand heat stress as they protect proteins from heat-induced damage (Farooq et al., 2011).

The gene structure analysis (Figure 3) identified several SNPs distinguishing tolerant and susceptible NILs located within the candidate genes, in which one marker wsnp_RFLContig2864_2688208 falls in the exon region. These markers can be used as functional markers, because not only does the exon encodes protein functional units but also noncoding DNA including intron and UTR has played significant roles in many studies (Cobb et al., 2008; Khurana et al., 2013; Lu et al., 2015; Grünewald et al., 2015). SNPs outside the identified candidate genes can still be used in marker-assisted selection for genetic and breeding research. Aharon et al. (2016) explored how far SNPs can be from the affected genes using a pathway-based approach and found that affected genes were often up to $2 \mathrm{Mbps}$ from the associated SNP and not necessarily the closest to the SNP.

The molecular mechanisms underlying heat tolerance in wheat remain unclear. A schematic network, proposed to explain the mechanism of heat tolerance in legumes, suggested that signalling and metabolic pathways, involving a series of physiochemical processes and important molecules such as HSPs, antioxidants, metabolites, and hormones, play key roles in regulating the legume response to heat stress (Liu et al., 2019). The candidate genes identified in this study are consistent, to a large extent, with their proposed network. Therefore, we hypothesize that such a pathway might exist in wheat, where many key genes collaboratively regulate the crop's response to heat stress (Figure 4).

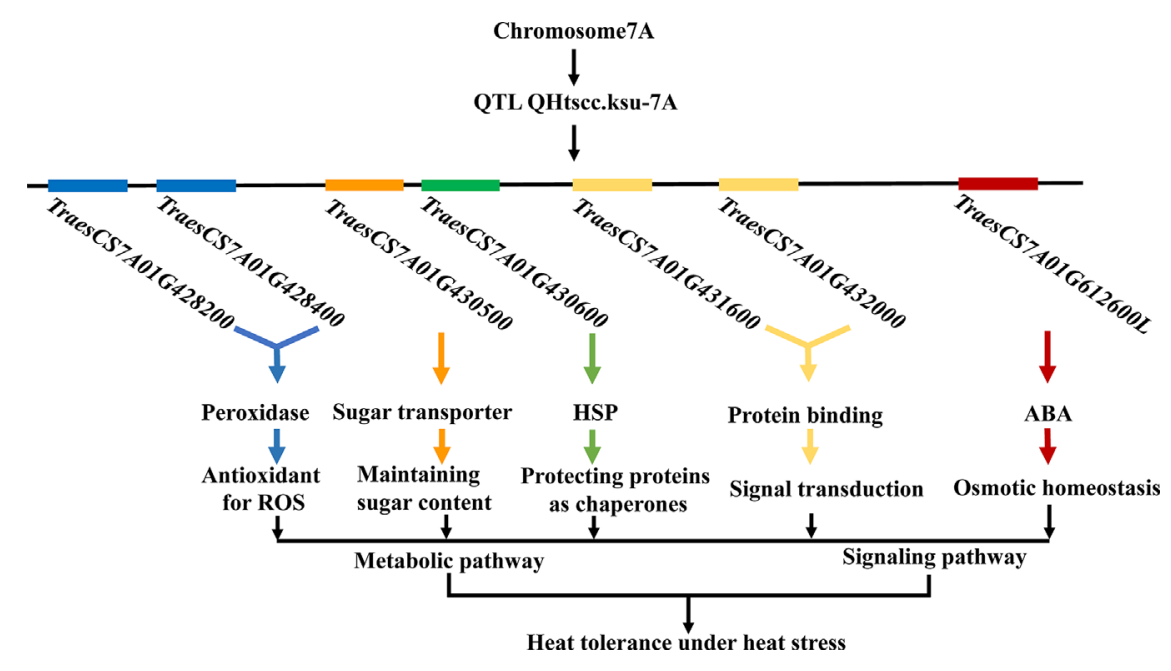

FIGURE 4 | Postulated pathway based on the findings of this study showing a collaborative regulation network of multiple genes in wheat in response to heat stress. Signaling pathway and metabolic pathway involving a series of physiochemical processes and important molecules, including HSPs, antioxidants, metabolites, and hormones. 


\section{CONCLUSIONS}

The NILs developed and validated in this study confirmed that the 7AL QTL, QHtscc.ksu-7A, is a major locus responsible for heat tolerance in wheat. The confirmed NILs and identified candidate genes are valuable resources for future studies in fine mapping and functional analyses of the chromosome region to clone the underlying gene(s).

\section{DATA AVAILABILITY STATEMENT}

All datasets presented in this study are included in the article/ supplementary material.

\section{AUTHOR CONTRIBUTIONS}

LL, HL, and GY conceived and designed the study. HL developed NIL populations, and LL conducted the experiments in the plant growth chambers and greenhouse. LL collected the relevant data and performed the analysis with guidance from GY and HL. LL prepared the manuscripts with inputs from YW, HL, and GY. All authors contributed to the article and approved the submitted version.

\section{REFERENCES}

Adams, J., Kelso, R., and Cooley, L. (2000). The kelch-repeat superfamily of proteins: propellers of cell function. Trends Cell Biol. 10 (1), 17-24. doi: 10.1016/S0962-8924(99)01673-6

Agarwal, P. K., and Jha, B. (2010). Transcription factors in plants and ABA dependent and independent abiotic stress signalling. Biol. Plant 54 (2), 201212. doi: 10.1007/s10535-010-0038-7

Aharon, B., Johnathan, R. A., and Yanay, O. (2016). How far from the SNP may the causative genes be? Nucleic Acids Res. 44 (13), 6046-6054. doi: 10.1093/nar/ gkw500

Alaux, M., Rogers, J., Letellier, T., Flores, R., Alfama, F., Pommier, C., et al. (2018). Linking the International Wheat Genome Sequencing Consortium bread wheat reference genome sequence to wheat genetic and phenomic data. Genome Biol. 19 (1), 111. doi: 10.1186/s13059-018-1491-4

Alsamadany, H. (2015). Diversity and genetic studies of heat tolerance in wheat. [Ph.D. thesis]. (Perth (WA): The University of Western Australia).

Araus, J. L., Amaro, T., Voltas, J., Nakkoul, H., and Nachit, M. M. (1998). Chlorophyll fluorescence as a selection criterion for grain yield in durum wheat under Mediterranean conditions. Field Crop Res. 55 (3), 209-223. doi: 10.1016/S0378-4290(97)00079-8

Avni, R., Nave, M., Eilam, T., Sela, H., Alekperov, C., Peleg, Z., et al. (2014). Ultradense genetic map of durum wheat $\times$ wild emmer wheat developed using the 90K iSelect SNP genotyping assay. Mol. Breed. 34 (4), 1549-1562. doi: 10.1007/ s11032-014-0176-2

Balla, K., Karsai, I., Bónis, P., Kiss, T., Berki, Z., Horváth, Á., et al. (2019). Heat stress responses in a large set of winter wheat cultivars (Triticum aestivum L.) depend on the timing and duration of stress. PloS One 14 (9), e0222639. doi: 10.1371/journal.pone.0222639

Baniwal, S. K., Bharti, K., Chan, K. Y., Fauth, M., Ganguli, A., Kotak, S., et al. (2004). Heat stress response in plants: a complex game with chaperones and more than twenty heat stress transcription factors. J. Biosci. 29, 471-487. doi: 10.1007/BF02712120

Blum, A., Klueva, N., and Nguyen, H. T. (2001). Wheat cellular thermotolerance is related to yield under heat stress. Euphytica 117 (2), 117-123. doi: 10.1023/ A:1004083305905

\section{FUNDING}

This research was funded by the Global Innovation Linkages Project (GIL53853) from the Australian Department of Industry, Innovation, and Science. The first author acknowledges the Research Training Program Scholarship from the Australian Government for sponsoring her Ph.D. study. The University of Western Australia provided funds for the open access publication fee.

\section{ACKNOWLEDGMENTS}

The authors would like to thank Professor Jacqueline Batley and Dr. Aneeta Pradhan for assistance in the 90k SNP array genotyping. The authors also thank Dr. Christine Davies at Tweak Editing for correcting English language errors.

\section{SUPPLEMENTARY MATERIAL}

The Supplementary Material for this article can be found online at: https://www.frontiersin.org/articles/10.3389/fpls.2020.01316/ full\#supplementary-material.

Budak, H., Kantar, M., Bulut, R., and Akpinar, B. A. (2015). Stress responsive miRNAs and isomiRs in cereals. Plant Sci. 235, 1-13. doi: 10.1016/ j.plantsci.2015.02.008

Cabral, A. L., Jordan, M. C., Larson, G., Somers, D. J., Humphreys, D. G., and McCartney, C. A. (2018). Relationship between QTL for grain shape, grain weight, test weight, milling yield, and plant height in the spring wheat cross RL4452/'ACDomain'. PloS One 13 (1), 1-32. doi: 10.1371/journal.pone. 0190681

Cavanagh, C. R., Chao, S. M., Wang, S. C., Huang, B. E., Stephen, S., Kiani, S., et al. (2013). Genome-wide comparative diversity uncovers multiple targets of selection for improvement in hexaploid wheat landraces and cultivars. Proc. Natl. Acad. Sci. U.S.A. 110 (20), 8057-8062. doi: 10.1073/pnas.1217133110

Caverzan, A., Casassola, A., and Brammer, S. P. (2016). Antioxidant responses of wheat plants under stress. Genet. Mol. Biol. 39 (1), 1-6. doi: 10.1590/16784685-GMB-2015-0109

Chen, D. Q., Wang, S. W., Cao, B. B., Cao, D., Leng, G. H., Li, H. B., et al. (2016). Genotypic variation in growth and physiological response to drought stress and re-watering reveals the critical role of recovery in drought adaptation in maize seedlings. Front. Plant Sci. 6, 1241. doi: 10.3389/fpls.2015.01241

Cobb, J., Büsst, C., Petrou, S., Harrap, S., and Ellis, J. (2008). Searching for functional genetic variants in non-coding DNA. Clin. Exp. Pharmacol. Physiol. 35 (4), 372-375. doi: 10.1111/j.1440-1681.2008.04880.x

Colasuonno, P., Gadaleta, A., Giancaspro, A., Nigro, D., Giove, S., Incerti, O., et al. (2014). Development of a high-density SNP-based linkage map and detection of yellow pigment content QTLs in durum wheat. Mol. Breed. 34 (4), 1563 1578. doi: 10.1007/s11032-014-0183-3

Craig, K. L., and Tyers, M. (1999). The F-box: a new motif for ubiquitin dependent proteolysis in cell cycle regulation and signal transduction. Prog. Biophys. Mol. Biol. 72 (3), 299-328. doi: 10.1016/S0079-6107(99)00010-3

Dawson, I. A., and Wardlaw, I. F. (1989). The tolerance of wheat to hightemperatures during reproductive growth. 3. Booting to anthesis. Aust. J. Agric. Res. 40 (5), 965-980. doi: 10.1071/Ar9890965

de Souza, R., Pena-Fleitas, M. T., Thompson, R. B., Gallardo, M., Grasso, R., and Padilla, F. M. (2019). The use of chlorophyll meters to assess crop N status and derivation of sufficiency values for sweet pepper. Sensors 19 (13), 1-20. doi: $10.3390 /$ s19132949 
Dixit, S., Kumar, B. A., Min, A., Henry, A., Oane, R. H., Raorane, M. L., et al. (2015). Action of multiple intra-QTL genes concerted around a co-localized transcription factor underpins a large effect QTL. Sci. Rep. 5, 15183-15183. doi: 10.1038/srep15183

Dorweiler, J., Stec, A., Kermicle, J., and Doebley, J. (1993). Teosinte-glumearchitecture-1- a genetic-locus controlling a key step in maize evolution. Science 262 (5131), 233-235. doi: 10.1126/science.262.5131.233

Driedonks, N., Rieu, I., and Vriezen, W. H. (2016). Breeding for plant heat tolerance at vegetative and reproductive stages. Plant Reprod. 29, 67-79. doi: 10.1007/s00497-016-0275-9

Farooq, M., Bramley, H., Palta, J. A., and Siddique, K. H. M. (2011). Heat stress in wheat during reproductive and grain-filling phases. Crit. Rev. Plant Sci. 30 (6), 491-507. doi: 10.1080/07352689.2011.615687

Fokar, M., Nguyen, H. T., and Blum, A. (1998). Heat tolerance in spring wheat. I. Estimating cellular thermotolerance and its heritability. Euphytica 104 (1), 1-8. doi: 10.1023/A:1018346901363

Gaju, O., DeSilva, J., Carvalho, P., Hawkesford, M. J., Griffiths, S., Greenland, A., et al. (2016). Leaf photosynthesis and associations with grain yield, biomass and nitrogen-use efficiency in landraces, synthetic-derived lines and cultivars in wheat. Field Crop Res. 193, 1-15. doi: 10.1016/j.fcr.2016.04.018

Galanti, R., Cho, A., Ahmad, A., and Mollinedo, J. (2019). Use of a chlorophyll meter for nondestructive and rapid estimation of leaf tissue nitrogen in macadamia. HortTechnology 29 (3), 308-313. doi: 10.21273/Horttech04276-19

Grünewald, T. G., Bernard, V., Gilardi-Hebenstreit, P., Raynal, V., Surdez, D., Aynaud, M. M., et al. (2015). Chimeric EWSR1-FLI1 regulates the Ewing sarcoma susceptibility gene EGR2 via a GGAA microsatellite. Nat. Genet. 47 (9), 1073-1078. doi: 10.1038/ng.3363

Haq, U. S., Khan, A., Ali, M., Khattak, A. M., Gai, W. X., Zhang, H. X., et al. (2019). Heat shock proteins: dynamic biomolecules to counter plant biotic and abiotic stresses. Int. J. Mol. Sci. 20:5321. doi: 10.3390/ijms20215321

Hefti, M. H., Francoijs, K. J., de Vries, S. C., Dixon, R., and Vervoort, J. (2004). The PAS fold-A redefinition of the PAS domain based upon structural prediction. Eur. J. Biochem. 271 (6), 1198-1208. doi: 10.1111/j.1432-1033.2004.04023.x

Hennig, S., Strauss, H. M., Vanselow, K., Yildiz, O., Schulze, S., Arens, J., et al. (2009). Structural and functional analyses of PAS domain interactions of the clock proteins drosophila period and mouse period2. PloS Biol. 7 (4), 836-853. doi: 10.1371/journal.pbio.1000094

IWGSC (2018). Shifting the limits in wheat research and breeding using a fully annotated reference genome. Science 361:7191. doi: 10.1126/science.aar7191

Khurana, E., Fu, Y., Colonna, V., Mu, X. J., Kang, H. M., Lappalainen, T., et al. (2013). Integrative annotation of variants from 1092 humans: application to cancer genomics. Science 342 (6154), 1235587. doi: 10.1126/science.1235587

Kovalic, D. K., Andersen, S. E., Byrum, J. R., Conner, T. W., Masucci, J. D., and Zhou, Y. (2007). Nucleic acid molecules and other molecules associated with plants and uses thereof for plant improvement (Washington, DC: U.S. Patent and Trademark Office).

Lalonde, S., Wipf, D., and Frommer, W. B. (2004). Transport mechanisms for organic forms of carbon and nitrogen between source and sink. Annu. Rev. Plant Biol. 55, 341-372. doi: 10.1146/annurev.arplant.55.031903.141758

Lemoine, R., La Camera, S., Atanassova, R., Deedaldeechamp, F., Allario, T., Pourtau, N., et al. (2013). Source-to-sink transport of sugar and regulation by environmental factors. Front. Plant Sci. 4, 272. doi: 10.3389/fpls.2013.00272

Li, C., Zhang, W., Yuan, M., Jiang, L., Sun, B., Zhang, D., et al. (2019). Transcriptome analysis of osmotic-responsive genes in ABA-dependent and -independent pathways in wheat (Triticum aestivum L.) roots. PeerJ 7:6519. doi: $10.7717 /$ peerj.6519

Liu, S., Zhang, X., Pumphrey, M. O., Stack, R. W., Gill, B. S., and Anderson, J. A. (2006). Complex microcolinearity among wheat, rice, and barley revealed by fine mapping of the genomic region harboring a major QTL for resistance to Fusarium head blight in wheat. Funct. Integr. Genomics 6 (2), 83-89. doi: 10.1007/s10142-005-0007-y

Liu, Y. X., Yang, X. M., Ma, J., Wei, Y. M., Zheng, Y. L., Ma, H. X., et al. (2010). Plant height affects fusarium crown rot severity in wheat. Phytopathology 100 (12), 1276-1281. doi: 10.1094/Phyto-05-10-0142

Liu, Y. C., Machuca, M. A., Beckham, S. A., Gunzburg, M. J., and Roujeinikova, A. (2015). Structural basis for amino-acid recognition and transmembrane signalling by tandem Per-Arnt-Sim (tandem PAS) chemoreceptor sensory domains. Acta Crystallogr. Sect. D-Struct. Biol. 71, 2127-2136. doi: 10.1107/\$139900471501384X
Liu, Y., Li, J., Zhu, Y., Jones, A., Ray, J. R., and Song, Y. (2019). Heat stress in legume seed setting: effects, causes, and future prospects. Front. Plant Sci. 10, 1-12. doi: 10.3389/fpls.2019.00938

Lu, Y. F., Mauger, D. M., Goldstein, D. B., Urban, T. J., Weeks, K. M., and Bradrick, S. S. (2015). IFNL3 mRNA structure is remodeled by a functional non-coding polymorphism associated with hepatitis C virus clearance. Sci. Rep. 5:16037. doi: $10.1038 /$ srep 16037

Manickavelu, A., Kawaura, K., Oishi, K., Shin-I, T., Kohara, Y., Yahiaoui, N., et al. (2012). Comprehensive functional analyses of expressed sequence tags in common wheat (Triticum aestivum). DNA Res. 19 (2), 165-177. doi: 10.1093/dnares/dss001

Marcum, K. B. (1998). Cell membrane thermostability and whole-plant heat tolerance of Kentucky bluegrass. Crop Sci. 38 (5), 1214-1218. doi: 10.2135/ cropsci1998.0011183X003800050017x

Mason, R. E., Mondal, S., Beecher, F. W., Pacheco, A., Jampala, B., Ibrahim, A. M. H., et al. (2010). QTL associated with heat susceptibility index in wheat (Triticum aestivum L.) under short-term reproductive stage heat stress. Euphytica 174 (3), 423-436. doi: 10.1007/s10681-010-0151-x

Mason, R. E., Mondal, S., Beecher, F. W., and Hays, D. B. (2011). Genetic loci linking improved heat tolerance in wheat (Triticum aestivum L.) to lower leaf and spike temperatures under controlled conditions. Euphytica 180 (2), 181194. doi: 10.1007/s10681-011-0349-6

Merchuk-Ovnat, L., Fahima, T., Krugman, T., and Saranga, Y. (2016). Ancestral QTL alleles from wild emmer wheat improve grain yield, biomass and photosynthesis across enviroinments in modern wheat. Plant Sci. 251, 23 34. doi: 10.1016/j.plantsci.2016.05.003

Mia, M. S., Liu, H., Wang, X. Y., and Yan, G. J. (2019). Multiple near-isogenic lines targeting a QTL hotspot of drought tolerance showed contrasting performance under post-anthesis water stress. Front. Plant Sci. 10, 271. doi: 10.3389/ fpls.2019.00271

Mirdita, V., Dhillon, B. S., Geiger, H. H., and Miedaner, T. (2008). Genetic variation for resistance to ergot (Claviceps purpurea [Fr.] Tul.) among full-sib families of five populations of winter rye (Secale cereale L.). Theor. Appl. Genet. 118 (1), 85-90. doi: 10.1007/s00122-008-0878-0

Moffatt, J. M., Sears, R. G., Cox, T. S., and Paulsen, G. M. (1990a). Wheat hightemperature tolerance during reproductive growth. II. genetic-analysis of chlorophyll fluorescence. Crop Sci. 30 (4), 886-889. doi: 10.2135/ cropsci1990.0011183X003000040025x

Moffatt, J. M., Sears, R. G., and Paulsen, G. M. (1990b). Wheat high temperature tolerance during reproductive growth. I. Evaluation by chlorophyll fluorescence. Crop Sci. 30, 881-885. doi: 10.2135/cropsci1990.0011183X003000040024x

Moglich, A., Ayers, R. A., and Moffat, K. (2009). Structure and signaling mechanism of Per-ARNT-Sim domains. Structure 17 (10), 1282-1294. doi: 10.1016/j.str.2009.08.011

Murray, M. G., and Thompson, W. F. (1980). Rapid isolation of high molecularweight plant DNA. Nucleic Acids Res. 8 (19), 4321-4325. doi: 10.1093/nar/ 8.19 .4321

Narayanan, S. (2018). Effects of high temperature stress and traits associated with tolerance in wheat. Open Access J. Sci. 2 (3), 177-186. doi: 10.15406/ oajs.2018.02.00067

Novichonok, E. V., Novichonok, A. O., Kurbatova, J. A., and Markovskaya, E. F. (2016). Use of the atLEAF plus chlorophyll meter for a nondestructive estimate of chlorophyll content. Photosynthetica 54 (1), 130-137. doi: 10.1007/s11099015-0172-8

Padilla, F. M., de Souza, R., Pena-Fleitas, M. T., Gallardo, M., Gimenez, C., and Thompson, R. B. (2018). Different responses of various chlorophyll meters to increasing nitrogen supply in sweet pepper. Front. Plant Sci. 9 (1752), 1-14. doi: $10.3389 /$ fpls.2018.01752

Paliwal, R., Roder, M. S., Kumar, U., Srivastava, J. P., and Joshi, A. K. (2012). QTL mapping of terminal heat tolerance in hexaploid wheat (T. aestivum L.). Theor. Appl. Genet. 125 (3), 561-575. doi: 10.1007/s00122-012-1853-3

Peng, J., Yu, D. S., Wang, L. Q., Xie, M. M., Yuan, C. Y., Wang, Y., et al. (2012). Arabidopsis F-box gene FOA1 involved in ABA signaling. Sci. China Life Sci. 55 (6), 497-506. doi: 10.1007/s11427-012-4332-9

Pietrini, F., Baccio, D. D., Iori, V., Veliksar, S., Lemanova, N., Juškaitè, L., et al. (2017). Investigation onmetal tolerance and phytoremoval activity in the poplar hybrid clone "Monviso" under Cu-spiked water: Potential use for wastewater treatment. Sci. Total Environ. 592, 412-418. doi: 10.1016/j.scitotenv.2017.03.101 
Pradhan, G. P., Prasad, P. V. V., Fritz, A. K., Kirkham, M. B., and Gill, B. S. (2012). Effects of drought and high temperature stress on synthetic hexaploid wheat. Func. Plant Biol. 39 (3), 190-198. doi: 10.1071/Fp11245

Prag, S., and Adams, J. C. (2003). Molecular phylogeny of the kelch-repeat superfamily reveals an expansion of $\mathrm{BTB} / \mathrm{kelch}$ proteins in animals. $B M C$ Bioinform. 4:42. doi: 10.1186/1471-2105-4-42

Priya, M., Dhanker, O. P., Siddique, K. H. M., HanumanthaRao, B., Nair, R. M., Pandey, S., et al. (2019). Drought and heat stress-related proteins: an update about their functional relevance in imparting stress tolerance in agricultural crops. Theor. Appl. Genet. 132 (6), 1607-1638. doi: 10.1007/s00122-019-03331-2

Pumphrey, M. O., Bernardo, R., and Anderson, J. A. (2007). Validating the Fhb1 QTL for fusarium head blight resistance in near-isogenic wheat lines developed from breeding populations. Crop Sci. 47 (1), 200-206. doi: 10.2135/ cropsci2006.03.0206

Rao, R. C. N., Talwar, H. S., and Wright, G. C. (2001). Rapid assessment of specific leaf area and leaf nitrogen in peanut (Arachis hypogaea L.) using a chlorophyll meter. J. Agron. Crop Sci. 186 (3), 175-182. doi: 10.1046/j.1439-037X.2001.00472.x

Raven, J. A., and Griffiths, H. (2015). Photosynthesis in reproductive structures: costs and benefits. J. Exp. Bot. 66 (7), 1699-1705. doi: 10.1093/jxb/erv009

Reynolds, M. P., Balota, M., Delgado, M.II, Amani, I., and Fischer, R. A. (1994). Physiological and morphological traits associated with spring wheat yield under hot, irrigated conditions. Aust. J. Plant Physiol. 21 (6), 717-730. doi: 10.1071/Pp9940717

Rezaei, E. E., Siebert, S., and Ewert, F. (2015). Intensity of heat stress in winter wheat-phenology compensates for the adverse effect of global warming. Environ. Res. Lett. 10:24012. doi: 10.1088/1748-9326/10/2/024012/meta

Rimbert, H., Darrier, B., Navarro, J., Kitt, J., Choulet, F., Leveugle, M., et al. (2018). High throughput SNP discovery and genotyping in hexaploid wheat. PloS One 13 (1), e0186329. doi: 10.1371/journal.pone.0186329

Russo, M. A., Ficco, D. B. M., Laido, G., Marone, D., Papa, R., Blanco, A., et al. (2014). A dense durum wheat $\mathrm{x}$ T-dicoccum linkage map based on SNP markers for the study of seed morphology. Mol. Breed. 34 (4), 1579-1597. doi: 10.1007/s11032-014-0181-5

Saadall, M. M., Quick, J. S., and Shanahan, J. F. (1990). Heat tolerance in winter wheat: II. membrane thermostability and field performance. Crop Sci. 30 (6), 1248-1251. doi: 10.2135/cropsci1990.0011183X003000060018x

Sairam, R. K., Deshmukh, P. S., and Saxena, D. C. (1998). Role of antioxidant systems in wheat genotypes tolerance to water stress. Biol. Plant 41, 387-394. doi: 10.1023/A:1001898310321

Shirdelmoghanloo, H., Taylor, J. D., Lohraseb, I., Rabie, H., Brien, C., Timmins, A., et al. (2016a). A QTL on the short arm of wheat (Triticum aestivum L.) chromosome 3B affects the stability of grain weight in plants exposed to a brief heat shock early in grain filling. BMC Plant Biol. 16, 100. doi: 10.1186/s12870-016-0784-6

Shirdelmoghanloo, H., Lohraseb, I., Rabie, H., Brien, C., and Timmins, A. (2016b). Heat susceptibility of grain filling in wheat (Triticum aestivum L.) linked with rapid chlorophyll loss during a 3-day heat treatment. Acta Physiol. Plant 38:208. doi: 10.1007/s11738-016-2208-5

Sukumaran, S., Dreisigacker, S., Lopes, M., Chavez, P., and Reynolds, M. P. (2015). Genome-wide association study for grain yield and related traits in an elite spring wheat population grown in temperate irrigated environments. Theor. Appl. Genet. 128 (2), 353-363. doi: 10.1007/s00122-014-2435-3

Suzuki, N., Miller, G., Morales, J., Shulaev, V., Torres, M. A., and Mittler, R. (2011). Respiratory burst oxidases: the engines of ROS signaling. Curr. Opin. Plant Biol. 14 (6), 691-699. doi: 10.1016/j.pbi.2011.07.014

Talukder, S. K., Babar, M. A., Vijayalakshmi, K., Poland, J., Prasad, P. V., Bowden, R., et al. (2014). Mapping QTL for the traits associated with heat tolerance in wheat (Triticum aestivum L.). BMC Genet. 15:97. doi: 10.1186/s12863-014-0097-4

Tao, Z. Q., Chen, Y. Q., Zou, J. X., Li, C., Yuan, S. F., Yan, P., et al. (2016). Spectral characteristics of spring maize varieties with different heat tolerance to high temperature. Spectrosc. Spectr. Anal. 36 (2), 520-526. doi: 10.3964/j.issn.10000593(2016)02-0520-07

Tuinstra, M. R., Ejeta, G., and Goldsbrough, P. B. (1997). Heterogeneous inbred family (HIF) analysis: a method for developing near-isogenic lines that differ at quantitative trait loci. Theor. Appl. Genet. 95 (5-6), 1005-1011. doi: 10.1007/s001220050654

van den Burg, H. A., Tsitsigiannis, D.II, Rowland, O., Lo, J., Rallapalli, G., MacLean, D., et al. (2008). The F-box protein ACRE189/ACIF1 regulates cell death and defense responses activated during pathogen recognition in tobacco and tomato. Plant Cell 20 (3), 697-719. doi: 10.1105/tpc.107.056978
Vasseur, F., Pantin, F., and Vile, D. (2011). Changes in light intensity reveal a major role for carbon balance in Arabidopsis responses to high temperature. Plant Cell Environ. 34 (9), 1563-1576. doi: 10.1111/j.1365-3040.2011. 02353.x

Vijayalakshmi, K., Fritz, A. K., Paulsen, G. M., Bai, G. H., Pandravada, S., and Gill, B. S. (2010). Modeling and mapping QTL for senescence-related traits in winter wheat under high temperature. Mol. Breed. 26 (2), 163-175. doi: 10.1007/s11032009-9366-8

Wahid, A., and Shabbir, A. (2005). Induction of heat stress tolerance in barley seedlings by pre-sowing seed treatment with glycinebetaine. Plant Growth Regul. 46 (2), 133-141. doi: 10.1007/s10725-005-8379-5

Wang, S. C., Wong, D. B., Forrest, K., Allen, A., Chao, S. M., Huang, B. E., et al. (2014). Characterization of polyploid wheat genomic diversity using a highdensity 90000 single nucleotide polymorphism array. Plant Biotechnol. 12 (6), 787-796. doi: 10.1111/pbi.12183

Wang, X. B., Wang, L. F., and Shangguan, Z. (2016). Leaf gas exchange and fluorescence of two winter wheat varieties in response to drought stress and nitrogen supply. PloS One 11 (11), ARTN e0165733. doi: 10.1371/journal.pone. 0165733

Wang, X., Liu, H., Mia, M. S., Siddique, K. H. M., and Yan, G. J. (2018). Development of near-isogenic lines targeting a major QTL on 3AL for preharvest sprouting resistance in bread wheat. Crop Pasture Sci. 69, 864-872. doi: $10.1071 / \mathrm{CP} 17423$

Wang, X., Liu, H., Liu, G., Mia, M. S., Siddique, K. H. M., and Yan, G. J. (2019). Phenotypic and genotypic characterization of near-isogenic lines targeting a major 4BL QTL responsible for pre-harvest sprouting in wheat. BMC Plant Biol. 19, 348. doi: 10.1186/s12870-019-1961-1

Wiegand, C. L., and Cuellar, J. A. (1981). Duration of grain filling and kernel weight of wheat as affected by temperature. Crop Sci. 21 (1), 95-101. doi: 10.2135/cropsci1981.0011183X001100010027x

Yan, G. J., Liu, H., Wang, H. B., Lu, Z. Y., Wang, Y. X., Mullan, D., et al. (2017) Accelerated generation of selfed pure line plants for gene identification and crop breeding. Front. Plant Sci. 8, 1-14. doi: 10.3389/fpls.2017.01786

Yang, J., Sears, R. G., Gill, B. S., and Paulsen, G. M. (2002). Growth and senescence characteristics associated with tolerance of wheat-alien amphiploids to high temperature under controlled conditions. Euphytica 126 (2), 185-193. doi: 10.1023/A:1016365728633

Yoshida, T., Mogami, J., and Yamaguchi-Shinozaki, K. (2014). ABA-dependent and ABA-independent signaling in response to osmotic stress in plants. Curr. Opin. Plant Biol. 21, 133-139. doi: 10.1016/j.pbi.2014.07.009

Zadoks, J. C. (1974). A decimal code for the growth stages of cereals. Weed Res. 14, 415-421. doi: 10.1111/j.1365-3180.1974.tb01084.x

Zhang, K., Liu, X. J., Ata-Ul-Karim, S. T., Lu, J. S., Krienke, B., Li, S. Y., et al. (2019). Development of chlorophyll-meter-index-based dynamic models for evaluation of high-yield japonica rice production in Yangtze River reaches. Agronomy-Basel 9 (2), 1-17. doi: 10.3390/agronomy9020106

Zhao, B., Ata-Ul-Karim, S. T., Liu, Z. D., Zhang, J. Y., Xiao, J. F., Liu, Z. G., et al. (2018). Simple assessment of nitrogen nutrition index in summer maize by using chlorophyll meter readings. Front. Plant Sci. 9 (11), 1-13. doi: 10.3389/ fpls.2018.00011

Zheng, Z., Wang, H. B., Chen, G. D., Yan, G. J., and Liu, C. J. (2013). A procedure allowing up to eight generations of wheat and nine generations of barley per annum. Euphytica 191 (2), 311-316. doi: 10.1007/s10681-013-0909-Z

Zhou, R., Kjaer, K. H., Rosenqvist, E., Yu, X., Wu, Z., and Ottosen, C. O. (2017). Physiological response to heat stress during seedling and anthesis stage in tomato genotypes differing in heat tolerance. J. Agron. Crop Sci. 203 (1), 68-80. doi: $10.1111 /$ jac. 12166

Conflict of Interest: The authors declare that the research was conducted in the absence of any commercial or financial relationships that could be construed as a potential conflict of interest.

Copyright (c) $2020 \mathrm{Lu}, \mathrm{Liu}, \mathrm{Wu}$ and Yan. This is an open-access article distributed under the terms of the Creative Commons Attribution License (CC BY). The use, distribution or reproduction in other forums is permitted, provided the original author(s) and the copyright owner(s) are credited and that the original publication in this journal is cited, in accordance with accepted academic practice. No use, distribution or reproduction is permitted which does not comply with these terms. 